\title{
Methylation-associated silencing of miR-128 promotes the development of esophageal cancer by targeting COX-2 in areas with a high incidence of esophageal cancer
}

\author{
JING JIN, TIANTIAN GUO, YONGDONG GUO, JIANGHUI LIU, FENG QU and YUTONG HE \\ Cancer Institute, Fourth Hospital of Hebei Medical University, Shijiazhuang, Hebei 050011, P.R. China
}

Received July 31, 2018; Accepted November 5, 2018

DOI: $10.3892 /$ ijo.2018.4653

\begin{abstract}
Esophageal cancer is one of the most common cancer types in the world, with a widely varying incidence between different regions. Zinc deficiency (ZD) is very common in high-risk areas for esophageal cancer. Dietary ZD is reported to be associated with esophageal squamous cell carcinoma (ESCC). In the current study, the effects of ZD on tumorigenesis and expression of inflammatory factors were investigated in mice. It was identified that a ZD diet advanced ESCC and increased the expression of cyclooxygenase-2 (COX-2) prior to the occurrence of ESCC in mice. ZD significantly enhanced DNA methyltransferase (DNMT) activity and increased the expression of DNMT1 and DNMT3B. Furthermore, the expression of miR-128 was downregulated by methylation, and COX-2, a direct target of miR-128, was upregulated with the reduction in miR-128. Upregulation of miR-128 inhibited the cell cycle, proliferation and metastasis, and the expression of COX-2, cyclin D1 and retinoblastoma protein $(\mathrm{Rb})$. Furthermore, the relative expression level of miR-128 was negatively associated with COX-2 in ESCC tissues. Collectively, these findings indicate that methylation-associated silencing of miR-128 promotes the development of esophageal cancer through upregulation of the expression of cyclin D1 and Rb by targeting COX-2 in ZD regions with a high incidence of esophageal cancer.
\end{abstract}

\section{Introduction}

Esophageal cancer, including esophageal squamous cell carcinoma (ESCC) and esophageal adenocarcinoma, is one of the most common cancer types in the world. It has the eighth highest incidence of all cancer types and the sixth highest cancer-associated mortality rate worldwide, based on GLOBOCAN 2012 (1). The incidence of esophageal cancer varies widely among different regions. For example,

Correspondence to: Professor Yutong He, Cancer Institute, Fourth Hospital of Hebei Medical University, 12 Jiankang Road, Shijiazhuang, Hebei 050011, P.R. China

E-mail: hytong69@yahoo.com

Key words: zinc deficiency, microRNA-128, cyclooxygenase-2, esophageal cancer, methylation it ranges from 3 per 100,000 in low incidence regions to over 100 per 100,000 in high-incidence areas (2). ESCC is the most common histological type of esophageal carcinoma in less economically developed countries, accounting for more than $95 \%$ of esophageal cancer cases (3). ESCC is typically diagnosed at an advanced stage because of the lack of early symptoms, usually resulting in poor prognosis, with an approximate 5-year overall survival rate of $20 \%$ (4). Thus, clarification of the pathogenic mechanisms and new methods for prevention are urgently needed.

Factors such as an underdeveloped economy, low nutrient intake and limited health resources are very common in high-risk areas of ESCC. Epidemiological studies have demonstrated that dietary zinc deficiency (ZD) is associated with the pathogenesis of ESCC (5). Abnet et al (6) indicated that zinc concentration measured by X-ray fluorescence in biopsy samples is negatively correlated with the subsequent risk of developing ESCC. Zinc is an essential trace element and a critical component of many enzymes (7).Zinc content is low in most foods, except for red meat and seafood, and people from high incidence areas of esophageal cancer whose primary diet does not include red meat and seafood are likely to be zinc-deficient. Zinc intake in high-incidence areas of esophageal cancer has been reported at only 72 and $62 \%$ of the recommended daily allowance in spring and autumn, respectively, and this may be one of the factors contributing to the high risk of esophageal cancer (8). Dietary ZD causes low serum zinc levels. Our previous study demonstrated that serum zinc levels in patients with ESCC were lower compared with those in healthy people and that serum zinc levels in people from areas with a high incidence of esophageal cancer were lower compared with those in people from low-incidence areas (9).

Dietary ZD has been demonstrated to promote ESCC development by inducing a distinct inflammatory signature. Epidemiological and clinical studies have indicated that chronic inflammation promotes the occurrence of cancer, including ESCC (10,11). Taccioli et al (12) reported that ZD amplifies the overexpression of pro-inflammatory mediators, including cyclooxygenase-2 (COX-2), S100a8 and S100a9 in the esophagus with accompanying esophageal epithelial hyperplasia in rats. This inflammatory signature is already activated in the early dysplastic stage. Taccioli et al (13) also demonstrated that ZD amplifies the inflammatory response to provide a microenvironment conducive to ESCC development. Additionally, supplementation with zinc reversed 
the inflammatory signature and prevented cancer formation. Thus, the molecular mechanism of ZD-induced inflammation has important clinical implications as a critical factor in ESCC development. The aim of the current study was to investigate the mechanism of ZD-induced inflammation and how it promotes esophageal cancer development.

\section{Materials and methods}

Ethical approval. The current study was approved by the Institutional Human Ethics Committee of Hebei Medical University Fourth Hospital (Shijiazhuang, China) and the Laboratory Animal Ethics Committee of Hebei Medical University Fourth Hospital. Written informed consent was obtained from all patients prior to their participation.

Animals and experimental design. A total of 120 6-week-old C57BL/6 mice (18 $\pm 2 \mathrm{~g}$; 1:1 male/female ratio) were obtained from Beijing Vital River Laboratory Animal Technology Co., Ltd. (Beijing, China). The ZD and zinc-sufficient (ZS) diets were identical with the exception of zinc content, which was 1.6 and $60.4 \mathrm{mg} / \mathrm{kg}$ (Beijing HFK Bioscience Co. Ltd. Beijing, China), respectively. The mice were randomly divided into two dietary groups (ZD and ZS, n=60 mice/group). All mice were treated with 4-Nitroquinoline 1-oxide (4NQO; Sigma-Aldrich; Merck KGaA, Darmstadt, Germany) in drinking water $(100 \mu \mathrm{g} / \mathrm{ml})$ from 6 weeks of age to establish an esophageal cancer model. The animals were weighed weekly and monitored daily. From each group, 6 mice were sacrificed at 16, 20, 24 and 32 weeks after chemical carcinogen treatment, and at 28 weeks, 36 mice were sacrificed in each group for tumor evaluation and gene expression profiling.

Human ESCC tissue samples. ESCC tissue and serum samples were obtained from 52 patients from Cixian, which is a region in Hebei province with a high incidence of histologically confirmed ESCC (2). Of the 52 patients, 38 were male $(73 \%)$ and 14 were female (27\%). The median age was 58 years old (range, 40-76 years old). The inclusion criterion was that the patient must have received a pathological diagnosis of primary ESCC. All patients were surgically treated at The Fourth Hospital of Hebei Medical University (also known as the Tumor Hospital of Hebei Province, which is a large and comprehensive level three, grade A hospital) from January 2011 to December 2012. All specimens were obtained within 30 min of surgery from each patient and immediately stored in liquid nitrogen; serum samples were collected prior to surgery. All patients were pathologically confirmed to have early-stage ESCC at the Hebei Medical University Fourth Hospital.

Immunohistochemical (IHC) assay. Human and mouse esophageal tissues were fixed in formalin and embedded in wax blocks. Paraffin sections ( $4 \mu \mathrm{m}$ thick) were deparaffinized and rehydrated, followed by treatment with $0.02 \mathrm{M}$ EDTA buffer (pH 9.0; Gene Tech, Shanghai, China). Then, the sections were immersed in $3 \% \mathrm{H}_{2} \mathrm{O}_{2}$ to quench endogenous peroxidase activity and blocked with 5\% normal goat serum (Zhongshanjingqiao Biotechnical Co., Ltd., Beijing, China), followed by incubation with a monoclonal anti-COX-2 antibody (\#12282, 1:250; Cell Signaling Technology, Inc., Danvers, MA, USA) overnight at $4^{\circ} \mathrm{C}$. The antibody was diluted in PBS buffer containing $5 \%$ normal goat serum. The negative control for each slide was incubated with 5\% normal goat serum without the anti-COX-2 antibody. The sections were then incubated with horseradish peroxidase-conjugated anti-rabbit IgG (cat. no. SP-9001, Zhongshanjingqiao Biotechnical Co., Ltd., Beijing, China) for $45 \mathrm{~min}$ at $37^{\circ} \mathrm{C}$ and visualized with diaminobenzidine tetrahydrochloride. The stained slides were examined under a light microscope and scored by three pathologists who were blinded to the clinical diagnosis. An index of COX-2 labeling was implemented so that samples were scored according to the percentage and intensity of staining among tumor cells (14).

Cell culture. The human esophageal cancer cell lines KYSE170 and Eca109, human esophageal epithelial cells NE1 and human embryonic kidney cells $293 \mathrm{~T}$ were donated by the MD Anderson CancerCenter,University of Texas(Houston,TX,USA).KYSE170 and Eca109 cells were cultured in RPMI-1640 medium (Hyclone; GE Healthcare, Logan, UT, USA) with $10 \%$ heat-inactivated fetal bovine serum (FBS; PAN-Biotech, Adenbach, Germany) at $37^{\circ} \mathrm{C}$ in a $5 \% \mathrm{CO}_{2}$ humidified incubator. NE1 and $293 \mathrm{~T}$ cells were cultured in Dulbecco's modified Eagle's medium (Gibco; Thermo Fisher Scientific, Inc., Waltham, MA, USA) with sodium pyruvate and $10 \%$ FBS. The membrane-permeable zinc chelator N,N,N',N'-tetrakis(2-pyridylmethyl)ethylenediamine (TPEN; Sigma-Aldrich; Merck KGaA) was prepared in ethanol to reduce zinc levels. Esophageal cancer cells were cultured with a gradient of different TPEN concentrations (from 0.001 to $0.011 \mu \mathrm{mol} / \mathrm{ml}$ in steps of $0.001 \mu \mathrm{mol} / \mathrm{ml}$ ) to study the effect of ZD on the expression level of COX-2. When the TPEN concentration increased to $0.011 \mu \mathrm{mol} / \mathrm{ml}$, the expression of COX-2 began to increase. ZD cells were cultured with $0.011 \mu \mathrm{mol} / \mathrm{ml}$ TPEN in subsequent experiments.

Cell-Counting-Kit 8 (CCK-8) assays. Esophageal cancer cells were seeded in 96 -well plates $\left(5 \times 10^{3}\right.$ cells/well). Proliferation was analyzed at 12, 24, 48 and $72 \mathrm{~h}$ with a simple test in which $10 \mu \mathrm{l}$ of CCK-8 (Solarbio Science and Technology Co., Ltd., Beijing, China) reagent was added to each well. The plates were then incubated at $37^{\circ} \mathrm{C}$ and $5 \% \mathrm{CO}_{2}$ for $2-4 \mathrm{~h}$, and the absorbance was recorded at $450 \mathrm{~nm}$.

Transwell assays. For the Transwell invasion assay, $2.5 \times 10^{4}$ cells were plated in the top chamber with a Matrigel-coated membrane (24-well insert; pore size, $8 \mu \mathrm{m}$; BD Biosciences, Franklin Lakes, NJ, USA). Cells were plated in RPMI-1640 medium modified without FBS, and medium supplemented with $10 \%$ FBS was used as a chemoattractant in the lower chamber. The cells were incubated for $24 \mathrm{~h}$, and cells that had not invaded through the pores were gently removed with a cotton swab. Cells on the lower surface of the membrane were fixed and stained with $0.5 \%$ Giemsa crystal violet solution (Solarbio Science and Technology Co., Ltd.) at room temperature for $15 \mathrm{~min}$ and counted under light microscopy.

Flow cytometry assays. Cells were harvested, washed twice with FBS and then fixed in a $70 \%$ ethanol solution for $24 \mathrm{~h}$ at $4^{\circ} \mathrm{C}$. Then, the fixed cells were washed once with PBS and resuspended as a single-cell suspension. The suspension was incubated with $500 \mu \mathrm{l}$ propidium iodide $(50 \mu \mathrm{g} / \mathrm{ml}$, Solarbio 
Table I. Primer sequences for quantitative polymerase chain reaction.

\begin{tabular}{lll}
\hline Name & \multicolumn{1}{c}{ Forward } & \multicolumn{1}{c}{ Reverse } \\
\hline miR-16 & CAGCCTAGCAGCACGTAAAT & GAGGTATTCGCACCAGAGGA \\
miR-128 & AACAAATATTAACACCTTCATACAACA & TGGTGTCGTGGAGTCG \\
miR-144 & GCGCGCTACAGTATAGATGATG & GCTGTCAACGATACGCTACG \\
miR-146a & GCAGGGTCCGAGGTATTCG & CGCGTGAGAACTGAATTCAT \\
RNU66 & GTGCTCGCTTCGGCAGCACATATAC & AAAAATATGGAACGCTCACGAATTG \\
COX-2 & GCTTTATGCTGAAGCCCTATGA & TCCAACTCTGCAGACATTTCC \\
CDK4 & GTTCGTGAGGTGGCTTTACT & ATGTCCTTAGGTCCTGGTCT \\
CDK6 & TCTTCCGTGTGAGTTGTTTG & TTGTGTGGCTCTATGTGTGC \\
Cyclin D1 & ATAATAAAGGGGTAATGGGG & GCGTTGTAGGAGAAAGGAAT \\
P53 & GGGTATCAAAGAAGGGCACT & ATTCAGCTTGGTTTACGGGC \\
Rb & CAAGGGTCATTATGGGTTAG & TTAGGTGTAGGGGAGGGGAG \\
\hline
\end{tabular}

miR, microRNA; CDK, cyclin-dependent kinase; COX-2, cyclooxygenase-2; Rb, retinoblastoma protein.

Science and Technology Co., Ltd.) for 30 min and evaluated using a flow cytometer.

Cell transfection. KYSE170 and Eca109 cells were plated $\sim 16 \mathrm{~h}$ prior to transfection. Hsa-miR-128 mimics were synthesized by Shanghai GeneChem Co., Ltd. (Shanghai, China), and the sequence of the mature miR-128 was 5'-UCA CAG UGA ACC GGU CUC UUU U-3'. Hsa-miR-128 mimics $(2 \mu \mathrm{g} / \mathrm{ml})$ were transfected into KYSE170 and Eca109 cells with Lipofectamine 2000 (Invitrogen; Thermo Fisher Scientific, Inc.). Hsa-miR-scramble was transfected into KYSE170 and Eca109 cells in the same way as a control, and the sequence of the scrambled miR was 5'-TGG ATC CAA GGT CGG GCA GGA AGA G-3'. The transfected cells were incubated for 4-6 h, and normal medium was added. The cells were harvested for further analysis after $48 \mathrm{~h}$.

Luciferasereporterassay.Cox2-associatedmiRNAs were queried via TargetScan (http://www.targetscan.org/mamm_31/) and The Cancer Genome Atlas (TCGA) database (https://cancergenome.nih.gov/). The predicted 3'-untranslated region (3'UTR) of COX-2, binding to miR-128, was cloned from the genomic DNA of esophageal cancer cells. The detailed sequence was as follows: GGT TGA ATG TTT GTC CTT AGG ATA GGC CTA TGT GCT AGC CCA CAA AGA ATA TTG TCT CAT TAG CCT GAA TGT GCC ATA AGA CTG ACC TTT TAA AAT GTT TTG AGG GAT CTG TGG ATG CTT CGT TAA TTT GTT CAG CCA CAA TTT ATT GAG AAA ATA TTC TGT GTC AAG CAC TGT GGG TTT TAA TAT TTT TAA ATC AAA CGC TGA TTA CAG ATA ATA GTA TTT ATA TAA ATA ATT GAA AAA AAT TTT CTT TTG GGA AGA GGG AGA AAA TGA AAT AAA TAT CAT TAA AGA TAA CTC AGG AGA ATC TTC TTT ACA ATT TTA CGT TTA GAA TGT T. The sequence was inserted into the pmirGLO control luciferase reporter vector (Shanghai GeneChem Co., Ltd.). The miR-128 mimics/blank vector and the wild-type/mutant COX-2 3'UTR were transfected into 293T cells and ECA109 cells with Lipofectamine 2000 (Shanghai GeneChem Co., Ltd.). Luciferase reporter assays were performed using the Dual-Glo luciferase assay system $24 \mathrm{~h}$ after transfection (GeneChem Co., Ltd., Shanghai, China).
Reverse transcription-quantitative polymerase chain reaction $(R T-q P C R)$. Total RNA was extracted from tissue samples, serum samples and esophageal cancer cells using the miRVana ${ }^{\mathrm{TM}}$ PARIS $^{\mathrm{TM}}$ kit (Ambion; Thermo Fisher Scientific, Inc.), according to the manufacturer's protocol. Total RNA for analysis of COX-2, CDK4, CDK6, cyclin D1 and p53 genes was extracted using TRIzol reagent (Invitrogen; Thermo Fisher Scientific, Inc.). RNA reverse transcription was performed with a RevertAid First Strand cDNA Synthesis kit (cat. no. K1622, Thermo Fisher Scientific, Inc.), for mRNA and an miRNAFirst Strand cDNA Synthesis kit (cat. no. B532453-0020, Sangon Biotech Co., Ltd., Shanghai, China) for miRNA. miRNA levels were analyzed using RT-qPCR with the miRcute Plus miRNA qPCR Detection kit (cat. no. FP411, Tiangen Biotech Co., Ltd., Beijing, China), and mRNA levels were analyzed with GoTaq ${ }^{\circledR}$ qPCR and RT-qPCR Systems (cat. no. A6001; Promega Corporation, Madison, WI, USA), SYBR Green and appropriate primers. Briefly, $10 \mathrm{ng}$ of total RNA was used as the template for $15 \mu l$ reverse transcription reactions. Probes were designed for specific mature miRNAs. For each miRNA, reactions were performed in triplicate using the 7500 RT-PCR system (Applied Biosystems; Thermo Fisher Scientific,Inc.), and RNU66 (Applied Biosystems; Thermo Fisher Scientific, Inc.; cat. no. 4427975) was used as the normalization control (9). Primer sequences are presented in Table I.

DNA methylation analysis. 5-aza-dCyd (Solarbio Science and Technology Co., Ltd.) treatment inhibits methylation of DNA. Genomic DNA from esophageal cancer tissue samples and cell lines was purified using DNAzol (Qiagen, Inc., Valencia, CA, USA). Sodium bisulfite conversion was conducted using a Qiagen Epitect Bisulfite kit (Qiagen, Inc.) according to the manufacturer's protocol. Methylation status of miR-128 was determined by methylation-specific PCR (MSP). PCR products were visualized by electrophoresis on a $2 \%$ agarose gel. The sequences of primers were as follows: Methylation-specific forward primer, 5'-TAG TAA AGC GAG AAT TTC GC-3' and reverse primer, 5'-CTA ACC GCC GAA AAT AAA C-3'; non-methylation-specific forward primer, 5'-GTA GTA AAG TGA GAA TTT TGT-3' and reverse primer, 5'-ACT AAC CAC CAA AAA TAA AC-3'. 
DNA methyltransferase (DNMT) activity. To estimate the activity of DNMTs, cells were collected and treated with a zinc concentration gradient (from 0.001 to $0.011 \mu \mathrm{mol} / \mathrm{ml}$ in steps of $0.001 \mu \mathrm{mol} / \mathrm{ml})$, then the cell pellet was obtained $(6,000 \mathrm{x} \mathrm{g}$, $\left.4^{\circ} \mathrm{C}, 5 \mathrm{~min}\right)$ and lysed by sonication $\left(12,000 \mathrm{~Hz}, 4^{\circ} \mathrm{C}, 5 \mathrm{~min}\right)$. The cells were then centrifuged at $13,200 \mathrm{x} \mathrm{g}$ for $30 \mathrm{~min}$ at $4^{\circ} \mathrm{C}$. The supernatant was collected, and the protein concentrations in cell lysates were determined with a Bradford reagent (Bio-Rad Laboratories, Inc., Hercules, CA, USA) using bovine serum albumin as the standard. The activity of DNMTs was determined using the EpiSeeker DNMT Activity Quantification Assay kit (Abcam, Cambridge, UK). In the assay, $5 \mu \mathrm{g}$ of cell extract was incubated with a universal DNMT substrate coated onto microplate wells. Methylated DNA was probed with detection antibodies provided with the kit. The amount of methylated DNA was colorimetrically quantified using ELISA to indicate enzyme activity (cat. no. CGE601Hu01, Wuhan USCN Business Co., Ltd., Wuhan, China).

Western blot analysis. Cells were lysed with ice-cold lysis buffer (Keygen Biotech Co.,Ltd., Nanjing, China) for 30 min on ice.Cell lysates were then collected after centrifugation at 13,200 $\mathrm{x}$ g for $5 \mathrm{~min}$ at $4^{\circ} \mathrm{C}$. Extracted protein concentration was determined by the BCA method. Lysate proteins $(60 \mu \mathrm{g})$ were loaded onto gels and separated by $12 \%$ SDS-PAGE, then transferred overnight at $4^{\circ} \mathrm{C}$ onto polyvinylidene difluoride membranes. Membranes were blocked with $0.05 \mathrm{~g} / \mathrm{ml}$ non-fat milk blocking solution for $1 \mathrm{~h}$ at room temperature. The membranes were incubated with antibodies [anti-COX-2 (cat. no. 12282), anti-cyclin D1 (cat.no. 3300) and anti-Rb (cat. no. 9309), 1:1,500, Cell Signaling Technology,Inc.; anti-DNMT1 (cat.no. ab13537), anti-DNMT3A (cat. no. ab2850) and anti-DNMT3B (cat. no. ab2851), 1:1,500, Abcam; anti-GAPDH (cat. no. G9545), 1:2,500, Sigma-Aldrich, Merck $\mathrm{KGaA}$ ) at $4^{\circ} \mathrm{C}$ overnight, washed three times with TBST with $0.1 \%$ Tween, and incubated with a horseradish peroxidase-linked secondary antibody (goat anti-rabbit, cat. no. 925-68071, 1:2,000, LI-COR Biosciences, Lincoln, NE, USA) for $1 \mathrm{~h}$ at room temperature. The membranes were washed three times with TBST with $0.1 \%$ Tween. The Odyssey imaging system (LI-COR Biosciences) was used to detect gray values.

Statistical analysis. All statistical analyses were performed using SPSS 13.0 software (SPSS Inc., Chicago, IL, USA). Quantitative results are presented as the mean \pm standard deviation. Comparisons of means between two groups was conducted using Student's t-test, while comparisons among more than two groups were conducted using one-way analysis of variance with Student-Newman-Keuls tests for pairwise comparisons. Pearson's correlation coefficient analysis was used to evaluate the correlation between miR-128 expression in tissue with COX-2 IHC scores or miR-128 in serum. $\mathrm{P}<0.05$ was considered to indicate a statistically significant difference.

\section{Results}

$Z D$ diet increases the incidence of esophageal cancer and increases the expression of $C O X-2$ in mice. Mice in the ZD and ZS groups were administered 4NQO to induce esophageal tumorigenesis. Histological examination revealed that esophageal cancer was first detected at 16 weeks in mice in the
ZD group (1/6) and at 20 weeks in mice in the ZS group (1/6; data not shown). The cut-off date was set as week 28 after 4NQO treatment. The incidence of esophageal cancer was significantly higher in mice in the $\mathrm{ZD}$ group compared with mice in the $\mathrm{ZS}$ group at week 28 (36/36 vs. 20/36; P<0.05; Fig. 1A).

To investigate the temporal and spatial localization of the key inflammation marker COX-2 in the ZD esophagus during cancer development, IHC was performed. Both the precancerous esophagus (16 weeks) and ESCC tissues (28 weeks) exhibited higher expression of COX-2 with the ZD diet compared with the $\mathrm{ZS}$ diet in mice (COX-2-positive rate: $4 / 6$ vs. $1 / 6, \mathrm{P}<0.05$ and $29 / 36$ vs. $11 / 36, P<0.05$, respectively). These results demonstrated that the expression of COX-2 increased due to ZD prior to the occurrence of esophageal cancer.

Downregulation of $m i R-128$ by $Z D$ increases the expression of $C O X-2$. To study the underlying mechanism by which COX-2 expression was increased by ZD, the public databases TargetScan and TCGA were searched. It was identified that the expression of COX-2 could be directly regulated by miR-128, miR-144 and miR-146a. The expression of COX-2 was evaluated in esophageal cancer cells cultured with different zinc levels regulated by TPEN concentration. The results indicated that the expression of COX-2 was increased by $54 \%$ when the concentration of TPEN was $0.011 \mu \mathrm{mol} / \mathrm{ml}$ $(\mathrm{P}<0.05$; Fig. 2A). Furthermore, the expression of miR-128, miR-144 and miR-146a were evaluated following treatment with $0.011 \mu \mathrm{mol} / \mathrm{ml} \mathrm{TPEN}$. It was identified that the expression of miR-128 $(\mathrm{P}<0.05)$, but not miR-144 or miR-146a, was downregulated, and the miR-128 expression level decreased by $22 \%$ compared with the control (Fig. 2B).

Expression of miR-128 in esophageal cancer tissues was significantly lower compared with paracarcinoma tissues and inversely related to the expression of $C O X-2$. miR-128 expression levels were examined by qRT-PCR and COX-2 expression level was evaluated by IHC in 52 pairs of early esophageal cancer and paracarcinoma tissues. Compared with the corresponding levels in paracarcinoma tissues, miR-128 expression level was upregulated in cancer tissues of $27 \%$ of patients, and downregulated in cancer tissues of $73 \%$ of patients. COX-2 expression was positive in $44 \%$ of adjacent tissues and $87 \%$ of cancer tissues. The relative expression level of miR-128 was negatively correlated with COX-2 expression as indicated by regression analysis $\left(\mathrm{R}^{2}=0.2749, \mathrm{~F}=18.954\right.$, $\mathrm{P}<0.05$; Fig. 3A). Relative miR-128 expression level in the serum was also detected using miR-16 as a standard and was observed to be positively correlated with miR-128 expression levels in tissues $\left(\mathrm{R}^{2}=0.4441, \mathrm{~F}=39.941, \mathrm{P}<0.05\right.$; Fig. 3B).

miR-128 is epigenetically silenced in esophageal cancer. As a possible mechanism of suppression of miR-128, the methylation status of $\mathrm{CpG}$ islands on promoter regions was evaluated with MSP. It was identified that the CpG islands of miR-128 were extensively methylated in esophageal cancer tumor samples and cell lines. The methylation level in esophageal cancer tissues was higher than that in paracarcinoma tissues $(38 / 52$ vs. 9/52; $\mathrm{P}<0.05$; Fig. 4A). To further determine whether the expression of miR-128 was silenced by DNA methylation, miR-128 expression was determined in esophageal cancer 

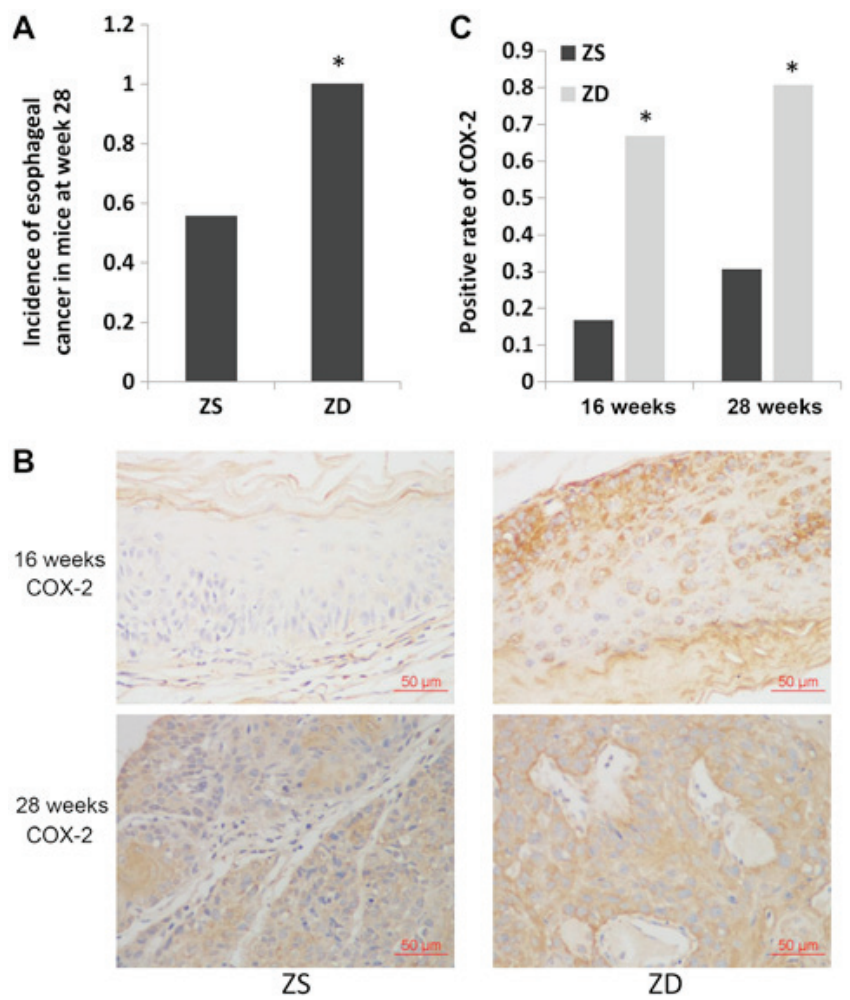

Figure 1. Zinc deficiency induces esophageal cancer and increases the expression of COX-2. (A) Incidence of esophageal cancer in mice on ZD and ZS diets at week 28 after $4 \mathrm{NQO}$ treatment. (B) Immunohistochemical analysis of COX-2 in the esophagus of mice on ZD and ZS diets at weeks 16 and 28 after $4 \mathrm{NQO}$ treatment. (C) COX-2-positive rate in the esophagus of mice on ZD and ZS diets a weeks 16 and 28 after 4 NQO treatment. " $\mathrm{P}<0.05$ vs. ZS. ZD, zinc-deficient; ZS, zinc-sufficient; COX-2, cyclooxygenase-2; 4NQO, 4-Nitroquinoline 1-oxide.
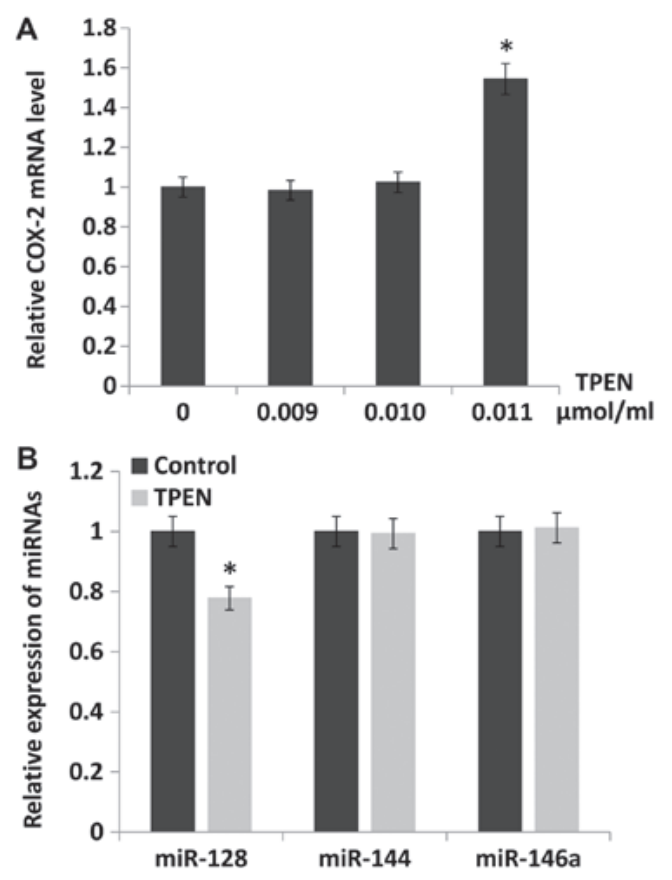

Figure 2. Effects of zinc deficiency on the expression of COX-2 and miRNAs. (A) Expression of COX-2 in esophageal cancer cells cultured with different zinc concentrations regulated by TPEN. ${ }^{*} \mathrm{P}<0.05 \mathrm{vs} .0 \mu \mathrm{mol} / \mathrm{ml}$. (B) Expression of miR-128, miR-144 and miR-146a in esophageal cancer cells cultured with $0.011 \mu \mathrm{mol} / \mathrm{ml}$ TPEN. "P $<0.05$ vs. Control. miR/miRNA, microRNA; COX-2, cyclooxygenase-2; TPEN, N,N,N',N'-tetrakis(2-pyridylmethyl)ethylenediamine.

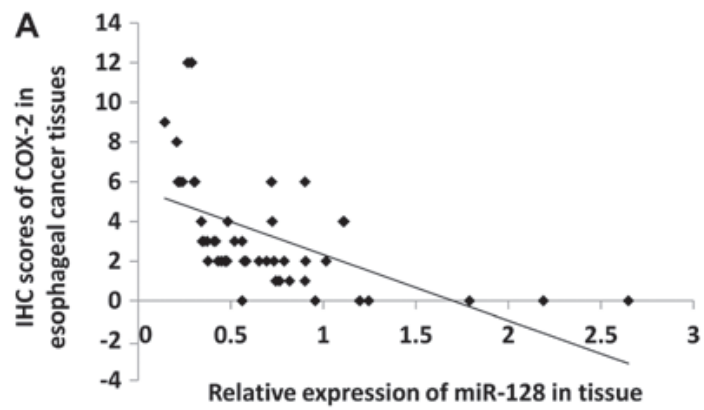

$Y=-3.3287 X+5.6577$

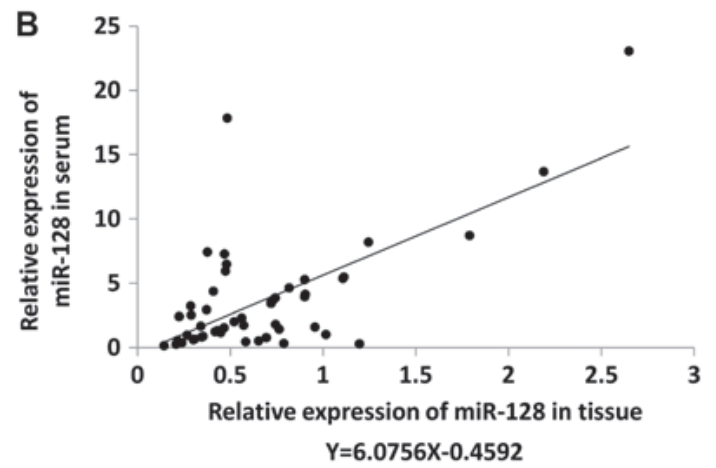

Figure 3. Associations between the relative expression of miR-128 and COX-2. (A) Association between relative expression of miR-128 and COX-2 in esophageal cancer tissues. (B) Association between the relative expression of miR-128 in esophageal cancer tissues and serum. miR, microRNA; COX-2, cyclooxygenase-2.
A

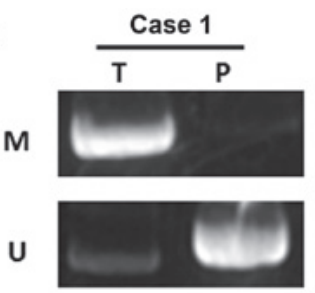

B
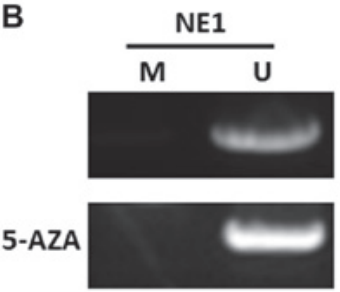
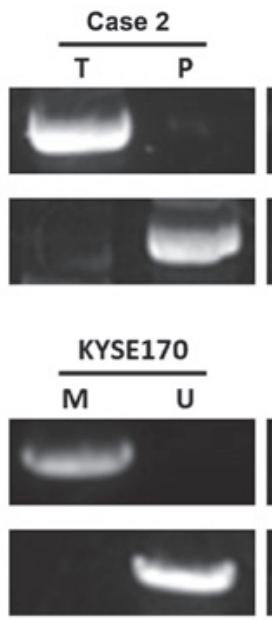
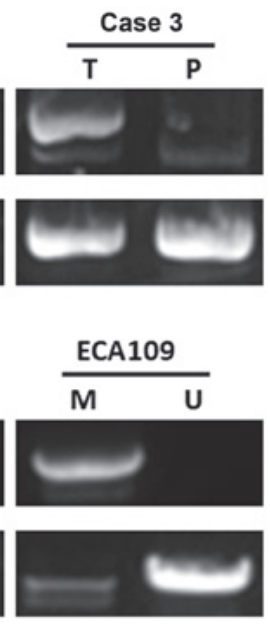

Figure 4. Methylation status of miR-128 CpG islands. (A) miR-128 methylation was detected by MSP in esophageal cancer and paracarcinoma tissues. (B) miR-128 methylation was detected by MSP with or without 5-AZA treatment in esophageal cancer cell lines. T, esophageal cancer tissue; $\mathrm{P}$, paracarcinoma tissue; MSP, methylation-specific polymerase chain reaction; M, MSP with methylation-specific primers; U, MSP with non-methylation-specific primers; 5-AZA, 5-aza-dCyd; miR, microRNA.

cell lines with or without 5 -aza-dCyd treatment. The results demonstrated that the miR-128 CpG islands were demethylated after treatment with 5-aza-dCyd, indicating that miR-128 was epigenetically silenced in esophageal cancer (Fig. 4B).

$Z D$ decreases miR-128 expression levels by increasing the methylation of miR-128 via enhancing DNMT activity. To 

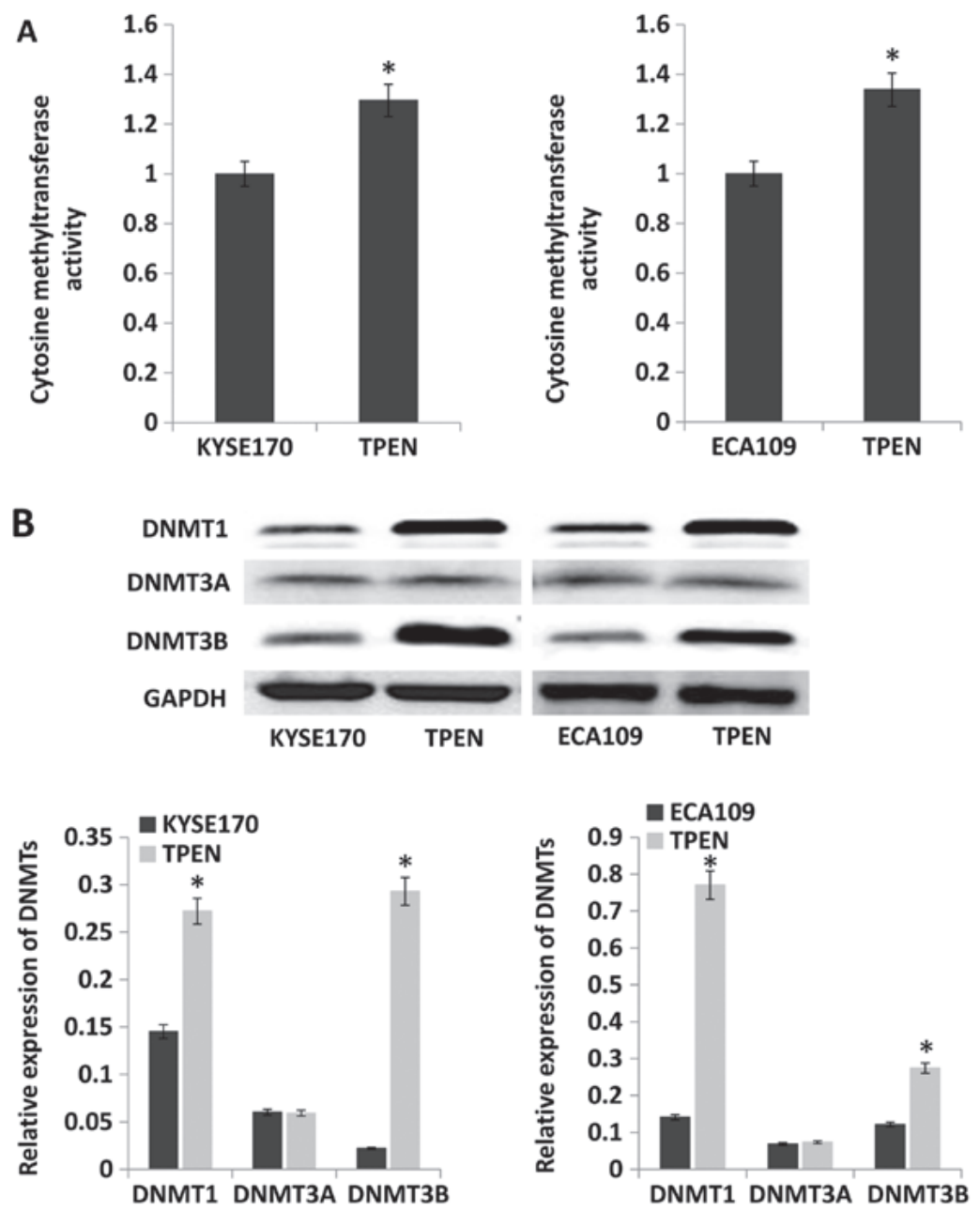

Figure 5. Activity and expression level of DNMTs. (A) Effect of TPEN on DNMT activity. (B) Changes in DNMT1, DNMT3A and DNMT3B expression evaluated by western blotting. " $\mathrm{P}<0.05$ vs. untreated cells. DNMT, DNA methyltransferase; TPEN, N,N,N',N'-tetrakis(2-pyridylmethyl)ethylenediamine.

examine the effect of ZD on DNMT activity, the activity and expression level of DNMTs was evaluated. The results indicated that compared with that in control cells, DNMT activity was significantly enhanced by $\sim 30$ and $34 \%$ in KYSE170 and Eca109 cells cultured with TPEN, respectively ( $\mathrm{P}<0.05$; Fig. 5A). Protein expression levels of DNMT1, DNMT3A and DNMT3B were evaluated by western blotting. The expression of DNMT1 and DNMT3B was increased by TPEN, but there was no difference in DNMT3A between cells cultured with and without TPEN (Fig. 5B).

Upregulation of miR-128 inhibits the proliferation and invasion of esophageal cancer cells. miR-128 mimics were transfected into the esophageal cancer cell lines KYSE170 and Eca109 to upregulate the expression of miR-128 (Fig. 6A). The proliferation of cell lines with miR-128 upregulation was significantly decreased compared with cells transfected with scrambled controls according to CCK-8 assay results $(\mathrm{P}<0.05)$. Proliferation was decreased by $35 \%$ in KYSE170 cells and 37\% in Eca109 cells (Fig. 6B and C). Furthermore, upregulation of miR-128 significantly decreased the invasion of esophageal cancer cells based on Transwell assays $(\mathrm{P}<0.05)$. Invasion was decreased by $35 \%$ in KYSE170 and 34\% in Eca109 cells (Fig. 6D and E). The results of flow cytometry analysis indicated that there was no significant difference in apoptosis rate following miR-128 upregulation in the two cell lines (apoptosis rate $<2 \%$ ). However, upregulation of miR-128 altered cell cycle distribution in esophageal cancer cells. The number of cells in G1 phase was increased by $20 \%$ in KYSE170 and 12\% in Eca109 cells (Fig. 6F and G).

Upregulation of miR-128 inhibits the expression of COX-2, cyclin DI and retinoblastoma protein $(R b)$. Cell cycle dysregulation is the main contributor to tumorigenesis (15). The expression of key cell cycle-related genes, cyclin-dependent kinase (CDK)4, CDK6, cyclin D1, P53, Rb and COX-2, was evaluated by RT-qPCR and western blotting. The results demonstrated that mRNA levels of COX-2, cyclin D1 and $\mathrm{Rb}$ were significantly lower in the miR-128 mimics group compared with the control group $(\mathrm{P}<0.05)$. However, the mRNA levels of CDK4, CDK6 and P53 were not significantly different between the groups (Fig. 7A and B). The protein levels of cyclin $\mathrm{D} 1, \mathrm{Rb}$ and $\mathrm{COX}-2$ were also detected, and the results were consistent with mRNA levels (Fig. 7C).

Upregulation of miR-128 directly inhibits the expression of COX-2. The databases TargetScan and miRanda were searched and it was identified that the seed sequence of miR-128 matched the 3 'UTR of COX-2 mRNA (Fig. 8A). Thus, miR-128 and luciferase reporter plasmids carrying the 3 'UTR of COX-2 

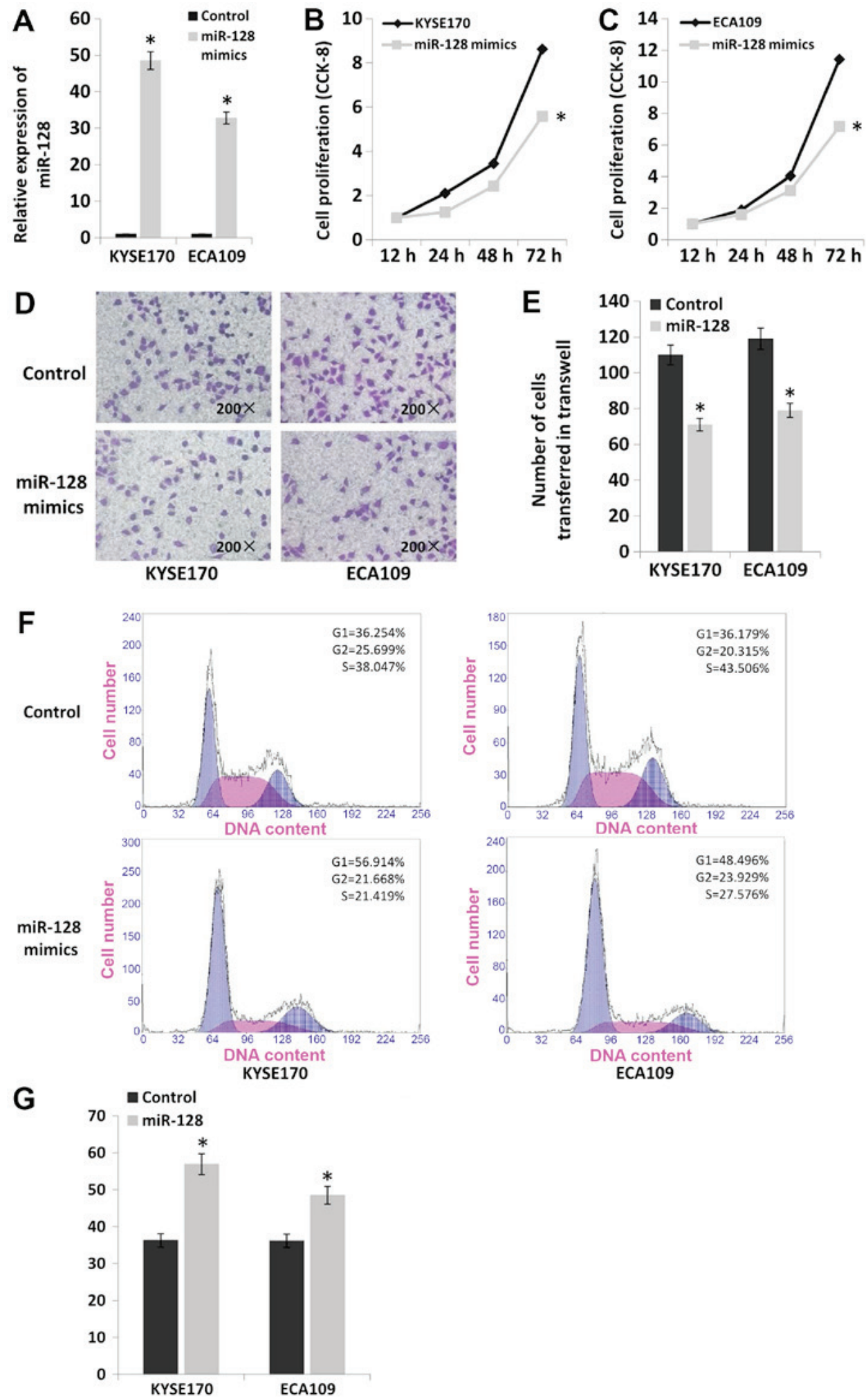

Figure 6. Upregulation of miR-128 suppresses cell proliferation and invasion in vitro. (A) miR-128 expression was detected in KYSE170 and Eca109 cells after transfection with miR-128 mimics or scrambled controls. (B and C) Cell proliferation was detected by Cell Counting Kit-8 assays after transfection of miR-128 mimics into KYSE170 and Eca109 cells. (D and E) Invasion was detected by Transwell assays after transfection of miR-128 mimics into KYSE170 and Eca109 cells. Magnification, x200. (F and G) Cell cycle distribution was detected by flow cytometry after transfection of miR-128 mimics into KYSE170 and Eca109 cells. ${ }^{*} \mathrm{P}<0.05$ vs. control. miR, microRNA.

containing the binding site of miR-128 were transfected into 293 T and ECA109 cells. Compared with the negative control, miR-128 overexpression significantly downregulated luciferase activity $(\mathrm{P}<0.05)$. However, miR-128 mimics did not alter the luciferase activity of the mutant construct without miR-128 binding sites (Fig. 8B). These results suggested that miR-128 directly suppressed COX-2 expression by targeting the 3'UTR of COX-2 mRNA. 
A

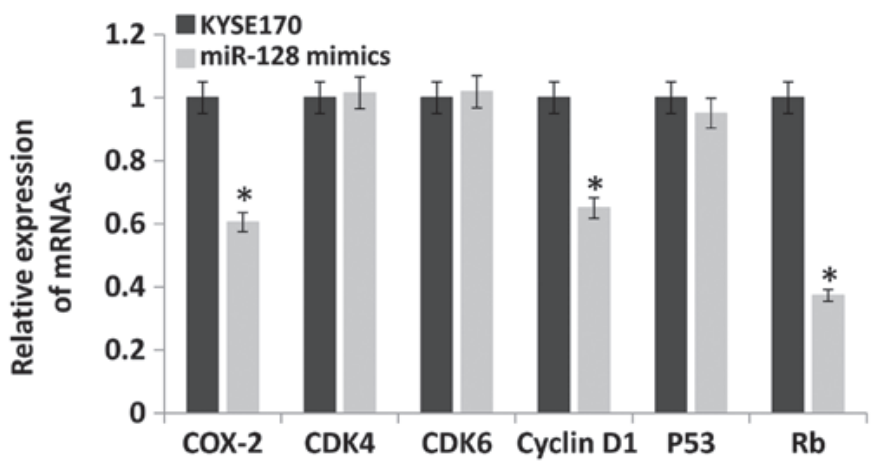

B

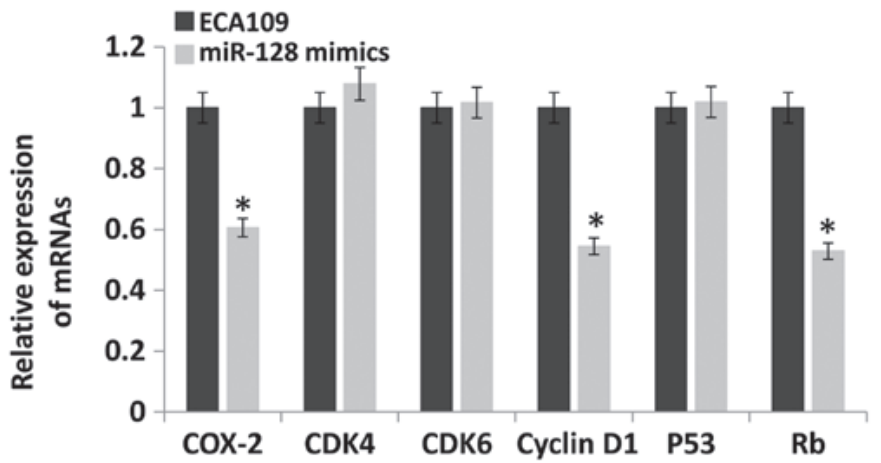

C
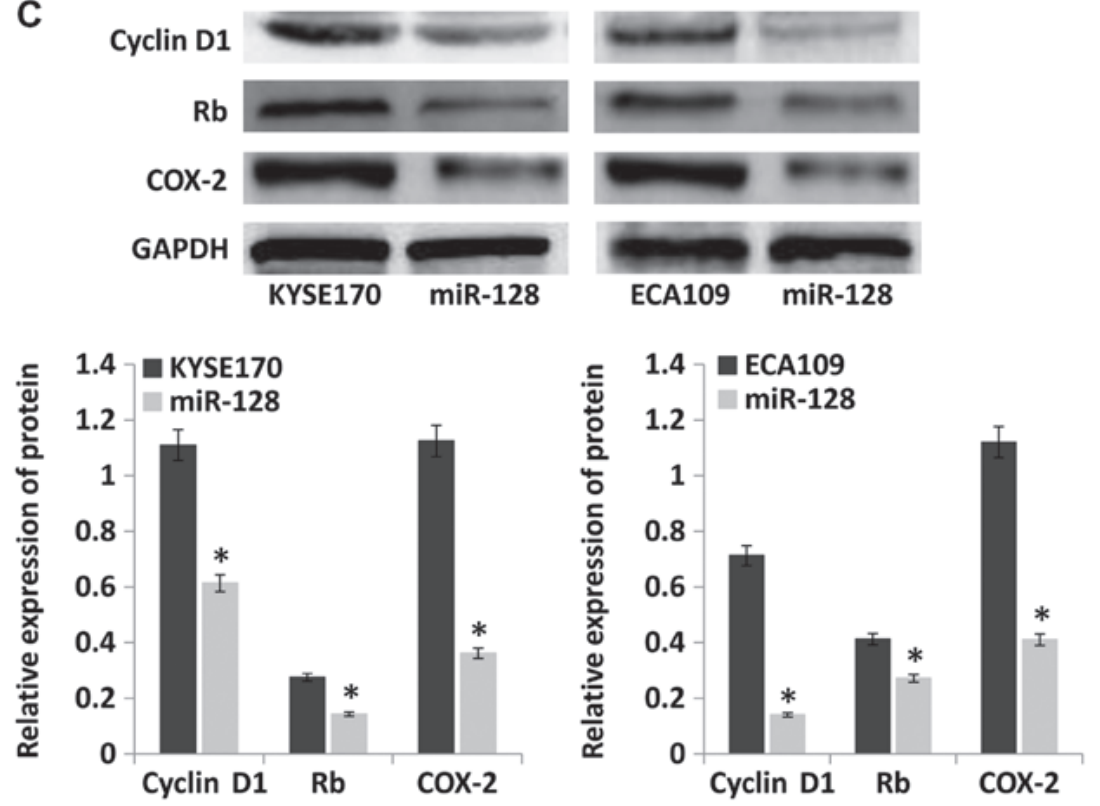

Figure 7. Upregulation of miR-128 inhibits the expression of COX-2, cyclin D1 and Rb. (A and B) mRNA expression of CDK4, CDK6, cyclin D1, P53, Rb and COX-2 was evaluated by RT-qPCR in KYSE170 and Eca109 cells after transfection with miR-128 mimics. (C) Protein expression of cyclin D1, Rb and COX-2 was evaluated by western blotting in KYSE170 and Eca109 cells after transfection with miR-128 mimics. "P<0.05 vs. control. miR, microRNA; CDK, cyclin-dependent kinase; COX-2, cyclooxygenase-2.

\section{Discussion}

The majority of esophageal cancer cases worldwide occur in the 'Asian esophageal cancer belt', which extends east from northern Iran to China (16). A well-known area for high incidence of esophageal cancer in China is the Taihang Mountains, which are situated in Hebei and Henan provinces. Cixian in Hebei province and its neighbor Linzhou in Henan province are the areas with the highest incidence of esophageal cancer in China and the world. The incidence of esophageal cancer in Cixian is 176.9 per 100,000 among men and 108.8 per 100,000 among women (world age-standardized incidence rate), which is much higher than the worldwide incidence (9.0 per 100,000 among men and 3.1 per 100,000 among women) (2). All of the patients in the current study were from Cixian. Studies in areas with a high incidence of esophageal cancer have implicated dietary ZD in the pathogenesis of ESCC $(5,9)$. Our previous study indicated that serum zinc levels in ESCC patients were lower compared with those in healthy people. Serum zinc levels in people from various high-incidence areas of ESCC were also significantly lower compared with those in people from 

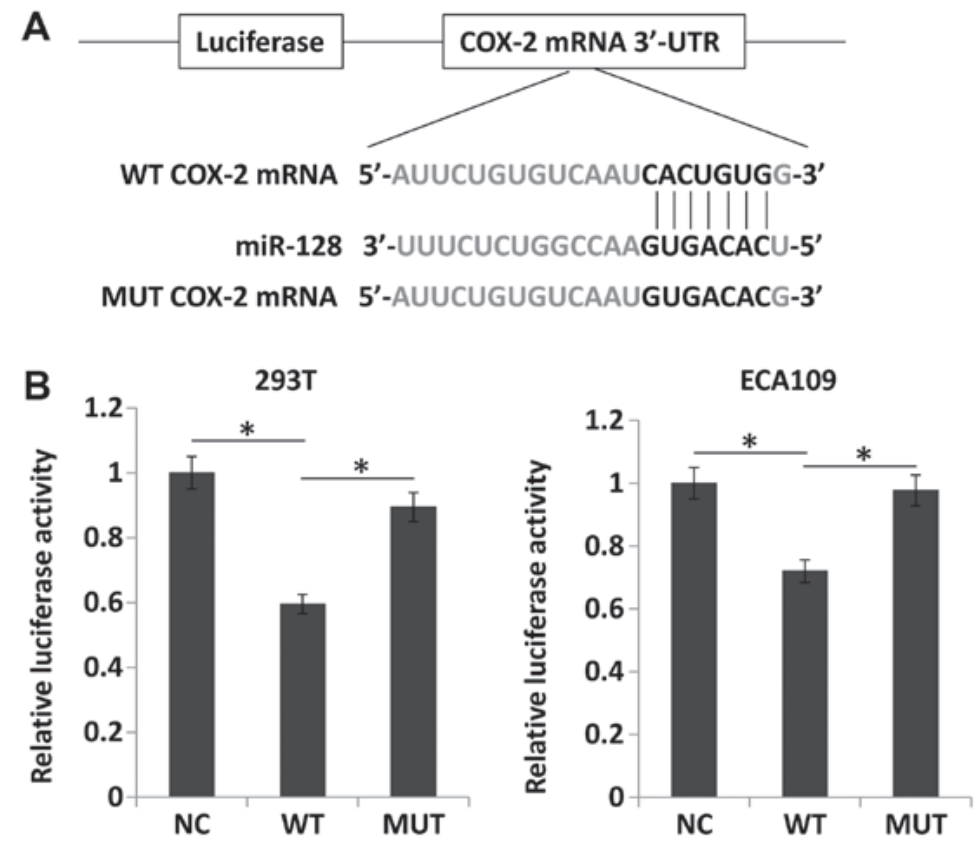

Figure 8 COX-2 is a direct target of miR-128. (A) Schematic diagram of COX-2 3'UTR and miR-128 binding site-containing reporter constructs. (B) Luciferase reporter assays in 293T and ECA109 cells following co-transfection of cells with blank plasmid, WT or MUT 3'UTR of COX-2 mRNA and miR-128, as indicated. "P<0.05. NC, negative control (blank plasmid); WT, wild-type; MUT, mutant; 3'UTR, 3'-untranslated region; miR, microRNA; COX-2, cyclooxygenase-2.

low-incidence areas (9). To study the role of ZD in esophageal cancer, mice were administered a ZD diet with $4 \mathrm{NQO}$ to establish an esophageal cancer model. The results indicated that ZD could promote the development of esophageal cancer. Certain inflammatory factors were also evaluated and it was identified that ZD could increase the expression of COX-2 prior to cancer initiation. These results suggested that ZD could facilitate esophageal cancer development by inducing certain inflammatory factors. These results are consistent with the conclusions of Taccioli et al (13).

The expression of inflammatory factors is regulated by various mechanisms, including the posttranscriptional regulation of gene expression by miRNAs. miRNAs are a family of short, noncoding RNAs that play an important role in coordinating complex programs of gene expression (17). miRNAs alter many biological processes, including cellular proliferation, apoptosis, immune response and signaling events (18). The inflamed ZD esophagus has a distinct miRNA signature that resembles human ESCC (19). Alder et al (20) demonstrated that chronic ZD induces an inflammatory gene signature that fuels ESCC development and induces a pro-tumorigenic miRNA signature. An inflamed ZD esophagus exhibits dysregulation of specific miRNAs resembling the miRNA signature of human ESCC, and zinc supplementation can prevent ESCC by correcting aberrant miRNA expression. Fong et al (21) also reported that miRNA dysregulation and ESCC progression depend on the extent of dietary ZD. Thus, ZD may promote the progression of esophageal cancer through regulation of the expression of inflammatory cytokines by changing the expression of miRNAs.

COX-2 is often induced during inflammation as a lipid mediator of inflammation (22). To study the mechanisms leading to the upregulated levels of COX-2 by ZD, in the current study the expression levels of miRNAs that directly regulate COX-2 were evaluated (23-25). The results indicated that miR-128 expression was decreased under ZD conditions, suggesting that the expression of COX-2 may be regulated by miR-128 under ZD conditions. Furthermore, the expression of miR-128 and COX-2 was evaluated in clinical specimens and it was identified that the expression of miR-128 in esophageal cancer tissues was negatively associated with COX-2 expression. This result was consistent with the results of the current in vitro experiments. Furthermore, the effect of ZD on the expression of miR-128 was evaluated. The results indicated that the expression of miR-128 was downregulated by methylation and that $\mathrm{ZD}$ increased the methylation of miR-128 by enhancing DNMT activity. DNA methylation can result in altered miRNA expression. Numerous tumor suppressor genes have already been identified to be methylated and transcriptionally silenced in cancers (26). miR-128 has been reported to be downregulated by DNA methylation in multiple cancers. For example, Yu et al (27) identified that miR-128 was downregulated by DNA methylation in gastric cancer and induced epithelial to mesenchymal transition through the P13K/AKT pathway. Takahashi et al (28) observed that miR-128 was silenced by DNA methylation in colorectal cancer and played an important role via the NEK2 pathway. In the current study, the role of miR-128 was evaluated in esophageal cancer in vitro and it was demonstrated that upregulation of miR-128 inhibited the proliferation and metastasis of esophageal cancer and increased the proportion of cells in interphase during cell division. Because cell cycle dysregulation is the main contributor to the occurrence of esophageal cancer, the expression of key cell cycle-associated genes was evaluated. The results demonstrated that the upregulation of miR-128 inhibited the expression of cyclin D1 and Rb. Cyclin D1 is 
a critical protein for the regulation of the G1 phase of the cell cycle, and $\mathrm{Rb}$ promotes the transition of cells from $\mathrm{G} 1$ to $S$ phase (29). Therefore, these data indicate that a decrease in miR-128 expression under ZD conditions promotes the occurrence of esophageal cancer by regulating cyclin D1 and $\mathrm{Rb}$ expression. COX-2 is a direct target of miR-128, and the expression levels of cyclin $\mathrm{D} 1$ and $\mathrm{Rb}$ are altered by COX-2 expression $(23,30,31)$. Thus, it is indicated that methylation-associated silencing of miR-128 promotes the development of esophageal cancer by upregulating the expression of cyclin D1 and Rb via targeting COX-2 in ZD areas with a high incidence of esophageal cancer.

The current study demonstrated the role of miR-128 and COX-2 in the early development of esophageal cancer and therefore their value as biomarkers for the early diagnosis of esophageal cancer could be considered. Although the expression levels of miR-128 and COX-2 in esophageal cancer tissues may reflect the progression of esophageal cancer, esophageal tissue specimens cannot be obtained during screening or diagnosis of early esophageal cancer. A good biomarker should be stable and easily obtainable. The expression of miRNAs in serum is stable and consistent with the expression of miRNAs in cancer tissues. For example, Yoshida et al (32) identified that miR-25 was highly expressed in the serum of patients with osteosarcoma and was also observed in patient tissues. Komatsu et al (33) reported that miRNAs are stably detectable in the serum and that circulating miRNAs may be tumor markers for ESCC. Further research has indicated that circulating miR-21 could be a useful biomarker for predicting chemoresistance (34). These results suggest that cancer may be diagnosable by detecting the levels of miRNAs in the blood. In the current study, the expression level of miR-128 in the serum of esophageal cancer patients was examined and was positively associated with miR-128 expression levels in tissues. Therefore, miR-128 expression levels in the serum may have potential use as a biomarker for the diagnosis of early esophageal cancer.

\section{Acknowledgements}

Not applicable.

\section{Funding}

This study was supported by grants from the National Natural Scientific Foundation of China (grant no. 81272682) and the Hebei Province Health Department (grant no. 20170745).

\section{Availability of data and materials}

The datasets used and/or analyzed during the current study are available from the corresponding author on reasonable request.

\section{Authors' contributions}

JJ performed cell experiments. TG performed animal experiments. YG analyzed the clinical specimens. JL performed flow cytometry. FQ performed immunohistochemistry analysis. YH provided theoretical guidance and supervised the study. All authors read and approved the final manuscript.

\section{Ethics approval and consent to participate}

The current study was approved by the Institutional Human Ethics Committee of Hebei Medical University Fourth Hospital (Shijiazhuang, China) and the Laboratory Animal Ethics Committee of Hebei Medical University Fourth Hospital. Written informed consent was obtained from all patients prior to their participation.

\section{Patient consent for publication}

Not applicable.

\section{Competing interests}

The authors declare that they have no competing interests.

\section{References}

1. Ferlay J, SI, Ervik M, Dikshit R, Eser S, Mathers C, Rebelo M, Parkin DM, Forman D and Bray F: GLOBOCAN 2012 v1.0, Cancer Incidence and Mortality Worldwide: IARC CancerBase No. 11, Lyon, France. International Agency for Research on Cancer; 2013. http://globocan.iarc.fr. Accessed December 12, 2013.

2. He J and Chen W: Chinese cancer registry annual report. Press Mil Med Sci Beijing 2012: 160-161, 2012.

3. Anvari K, Sima HR, Seilanian Toussi M, Anvari A, Shahidsales S, Memar B, Aledavoud SA, Forghani MN, Abdollahi A and Ghaffarzadegan K: EGFR expression in patients with esophageal squamous cell carcinoma and its association with pathologic response to preoperative chemoradiotherapy: A study in Northeastern Iran. Arch Iran Med 20: 240-245, 2017.

4. Zeng H, Zheng R, Guo Y, Zhang S, Zou X, Wang N, Zhang $\mathrm{L}$, Tang J, Chen J, Wei K, et al: Cancer survival in China, 2003-2005: A population-based study. Int J Cancer 136: 1921-1930, 2015.

5. Hashemian M, Poustchi H, Abnet CC, Boffetta P, Dawsey SM, BrennanPJ,Pharoah P,Etemadi A,KamangarF, Sharafkhah M, et al: Dietary intake of minerals and risk of esophageal squamous cell carcinoma: Results from the Golestan Cohort Study. Am J Clin Nutr 102: 102-108, 2015.

6. Abnet CC, Lai B, Qiao YL, Vogt S, Luo XM, Taylor PR, Dong ZW, Mark SD and Dawsey SM: Zinc concentration in esophageal biopsy specimens measured by X-ray fluorescence and esophageal cancer risk. J Natl Cancer Inst 97: 301-306, 2005.

7. Gaither LA and Eide DJ: Eukaryotic zinc transporters and their regulation. Biometals 14: 251-270, 2001.

8. Zou XN, Taylor PR, Mark SD, Chao A, Wang W, Dawsey SM, Wu YP, Qiao YL and Zheng SF: Seasonal variation of food consumption and selected nutrient intake in Linxian, a high risk area for esophageal cancer in China. Int J Vitam Nutr Res 72: 375-382, 2002.

9. He Y, Jin J, Wang L, Hu Y, Liang D, Yang H, Liu Y and Shan B: Evaluation of miR-21 and miR-375 as prognostic biomarkers in oesophageal cancer in high-risk areas in China. Clin Exp Metastasis 34: 73-84, 2017.

10. Balkwill F and Mantovani A: Inflammation and cancer: back to Virchow? Lancet 357: 539-545, 2001.

11. Coussens LM and Werb Z: Inflammation and cancer. Nature 420: 860-867, 2002.

12. Taccioli C, Wan S-G, Liu C-G, Alder H, Volinia S, Farber JL, Croce CM and Fong LY: Zinc replenishment reverses overexpression of the proinflammatory mediator S100A8 and esophageal preneoplasia in the rat. Gastroenterology 136: 953-966, 2009.

13. Taccioli, H Chen, Y Jiang, Liu XP, Huang K, Smalley KJ, Farber JL, Croce CM and Fong LY: Dietary zinc deficiency fuels esophageal cancer development by inducing a distinct inflammatory signature. Oncogene 31: 4550-4558, 2012.

14. Jin J, Li Z, Liu J, Wu Y, Gao X and He Y: Knockdown of zinc transporter ZIP5 (SLC39A5) expression significantly inhibits human esophageal cancer progression. Oncol Rep 34: 1431-1439, 2015. 
15. Zhao S, Jiang Y, Zhao J, Li H, Yin X, Wang Y, Xie Y, Chen X, Lu J, Dong Z, et al: Quercetin-3-methyl ether inhibits esophageal carcinogenesis by targeting the AKT/mTOR/p70S6K and MAPK pathways. Mol Carcinog 57: 1540-1552, 2018.

16. Cortés González R and Villaseñor Caloca R: Esophageal cancer. Rev Gastroenterol Mex 62: 149-159, 1997 (In Spanish).

17. Ambros V: MicroRNA pathways in flies and worms: Growth, death, fat, stress, and timing. Cell 113: 673-676, 2003.

18. Bartel DP: MicroRNAs: Target recognition and regulatory functions. Cell 136: 215-233, 2009.

19. Fong LY, Taccioli C, Jing R, Smalley KJ, Alder H, Jiang Y, Fadda P, Farber JL and Croce CM: MicroRNA dysregulation and esophageal cancer development depend on the extent of zinc dietary deficiency. Oncotarget 7: 10723-10738, 2016.

20. Alder H, Taccioli C, Chen H, Jiang Y, Smalley KJ, Fadda P, Ozer HG, Huebner K, Farber JL, Croce CM, et al: Dysregulation of miR-31 and miR-21 induced by zinc deficiency promotes esophageal cancer. Carcinogenesis 33: 1736-1744, 2012

21. Fong LY, Taccioli C, Jing R, Smalley KJ, Alder H, Jiang Y, Fadda P, Farber JL and Croce CM: MicroRNA dysregulation and esophageal cancer development depend on the extent of zinc dietary deficiency. Oncotarget 7: 10723-1038, 2016.

22. Grosser T, Yu Y and Fitzgerald GA: Emotion recollected in tranquility: Lessons learned from the COX-2 saga. Annu Rev Med 61: 17-33, 2010

23. Lin Y and Wu Z: MicroRNA-128 inhibits proliferation and invasion of glioma cells by targeting COX-2. Gene 658: 63-69, 2018.

24. Yao Q, Gu A, Wang Z and Xue Y: MicroRNA-144 functions as a tumor suppressor in gastric cancer by targeting cyclooxygenase-2. Exp Ther Med 15: 3088-3095, 2018.

25. Cornett AL and Lutz CS: Regulation of COX-2 expression by miR-146a in lung cancer cells. RNA 20: 1419-1430, 2014.

26. Espinosa-Parrilla Y, Muñoz X, Bonet C, Garcia N, Venceslá A Yiannakouris N, Naccarati A, Sieri S, Panico S, Huerta JM, et al: Genetic association of gastric cancer with miRNA clusters including the cancer-related genes MIR29, MIR25, MIR93 and MIR106: Results from the EPIC-EURGAST study. Int J Cancer 135: 2065-2076, 2014.
27. Yu WW, Jiang H,Zhang CT and Peng Y: The SNAIL/miR-128 axis regulated growth, invasion, metastasis, and epithelial-to-mesenchymal transition of gastric cancer. Oncotarget 8: 39280-39295, 2017.

28. Takahashi Y, Iwaya T, Sawada G, Kurashige J, Matsumura T, Uchi R, Ueo H, Takano Y, Eguchi H, Sudo T, et al: Up-regulation of NEK 2 by microRNA-128 methylation is associated with poor prognosis in colorectal cancer. Ann Surg Oncol 21: 205-212, 2014.

29. Feng Z, Xia Y, Gao T, Xu F1, Lei Q1, Peng C2, Yang Y3, Xue Q1, $\mathrm{Hu} \mathrm{X} 1$, Wang Q, et al: The antipsychotic agent trifluoperazine hydrochloride suppresses triple-negative breast cancer tumor growth and brain metastasis by inducing G0/G1 arrest and apoptosis. Cell Death Dis 9: 1006, 2018.

30. Zhuang ZH, Tsao SW, Deng W, Wang JD, Xia HH, He H, Feng HC, Wang LD, Gu Q, Lam SK, et al: Early upregulation of cyclooxygenase- 2 in human papillomavirus type 16 and telomerase-induced immortalization of human esophageal epithelial cells. J Gastroenterol Hepatol 23: 1613-1620, 2008.

31. Liu B, Wen JK, Li BH, Fang XM, Wang JJ, Zhang YP, Shi CJ, Zhang DQ and Han M: Celecoxib and acetylbritannilactone interact synergistically to suppress breast cancer cell growth via COX-2-dependent and -independent mechanisms. Cell Death Dis 28: e185, 2011

32. Yoshida A, Fujiwara T, Uotani K, Morita T, Kiyono M, Yokoo S, Hasei J, Nakata E, Kunisada T and Ozaki T: Clinical and functional significance of intracellular and extracellular microRNA-25-3p in osteosarcoma. Acta Med Okayama 72: $165-174,2018$.

33. Komatsu S, Ichikawa D, Takeshita $H$, Tsujiura M, Morimura $\mathrm{R}$, Nagata H, Kosuga T, Iitaka D, Konishi H, Shiozaki A, et al: Circulating microRNAs in plasma of patients with oesophageal squamous cell carcinoma. Br J Cancer 105: 104-111, 2011.

34. Komatsu S, Ichikawa D, Kawaguchi T, Miyamae M, Okajima W, Ohashi T, Imamura T, Kiuchi J, Konishi H, Shiozaki A, et al: Circulating miR-21 as an independent predictive biomarker for chemoresistance in esophageal squamous cell carcinoma. Am J Cancer Res 6: 1511-1523, 2016. 